Paula Andrea Nieto Alemán* Jaime Alberto Rendón Acevedo*"

Recibido: 14 de agosto de 2018 Concepto de evaluación: 5 de diciembre de 2018

Aprobado: 8 de febrero de 2019

Artículo de investigación (C) 2019 Universidad Católica de Colombia. Facultad de Ciencias Económicas y Administrativas. Todos los derechos reservados

* Doctora en Economía Agroalimentaria. Docente investigadora del Departamento de Economía y Ciencias Sociales de la Universitat Politècnica de València y profesora asociada de la Universidad Internacional de València. España. Correo electrónico: paunieal@upv.es (D) https://orcid.org/0000-0002-9269-5238

** Doctor en Economía Internacional y Desarrollo. Profesor e investigador de la Facultad de Ciencias Económicas y Sociales, Universidad de La Salle. Colombia. Correo electrónico: jrendon@unisalle.edu.co (D) https://orcid.org/0000-0001-8726-3759
Finanz. polit. econ., ISSN: 2248-6046, Vol. 11, N. ${ }^{\circ}$ 1, enero-junio, 2019, pp. 23-39

http://doi.org/10.14718/revfinanzpolitecon.2019.11.1.2

\section{¿Es la integración regional un vehículo para la convergencia? El caso del Mercosur, 1990-2014}

\section{RESUMEN}

Este artículo responde a dos cuestionamientos: a) iel proceso de integración regional en el Mercosur, desde su lanzamiento con el Tratado de Asunción (1991), ha contribuido a la reducción de las disparidades en el ingreso per cápita entre los países de la región?, b) ise ha dado convergencia vía el comercio intrazona? Para responderlos, fue necesario revisar el marco teórico de la integración y buscar explicaciones que justificaran el proceso de integración en el Mercosur bajo el esquema de unión aduanera y su vinculación con la convergencia. Se entiende la integración como un proceso económico influenciado por las estructuras productivas de los países y cuyo patrón de comercio responde a la asignación de recursos de la estructura sectorial de la producción. Utilizando el modelo por efectos fijos, se observó que las regresiones no evidencian existencia de $\beta$-convergencia en el PIB per cápita del conjunto de países. Adicionalmente, se estimó un modelo en el que se incluye el índice de apertura global y el índice de apertura intrarregional. Los resultados no revelan presencia de $\beta$-convergencia condicional para el conjunto de países. Se puede concluir que el Mercosur no ha favorecido el crecimiento de los países en términos de PIB per cápita.

Palabras clave: convergencia y divergencia regional, integración regional, unión aduanera, Mercosur.

JEL: F11, F15, O47.

Cómo citar este artículo / To reference this article / Para citar este artigo:

Nieto Alemán, P., \& Rendón Acevedo, J. (2019). ¿Es la integración regional un vehículo para la convergencia? El caso del Mercosur, 1990-2014. Revista Finanzas y Política Económica, 11 (1), 23-39. doi:http://dx.doi.org/10.14718/http://doi.org/10.14718/ revfinanzpolitecon.2019.11.1.2

\section{Is regional integration a vehicle for convergence? The case of Mercosur, 1990-2014}

\section{ABSTRACT}

This article seeks to answer two questions: Has the process of regional integration in Mercosur since its launch with the Treaty of Asunción (1991) contributed to reducing disparities in per capita income among countries in 
the region? Has there been convergence via intra-zone trade? To do this, it was necessary to review the theoretical framework of integration and to seek explanations that would justify the Mercosur integration process under the customs union scheme and its link with convergence. Integration is understood here as an economic process influenced by the productive structures of countries, whose pattern of trade responds to the allocation of resources of the sectoral structure of production. Using the fixed effects model, it was observed that regressions do not show the existence of $\beta$-convergence in the GDP per capita of the set of countries. Additionally, a model was estimated based on the global open data index and intra-regional openness index. The results do not reveal conditional $\beta$-convergence for the set of countries. The paper concludes that Mercosur has not favored the growth of countries in terms of GDP per capita.

Keywords: Mercosur, regional convergence and divergence, regional integration, customs union.

\section{A integração regional é um veículo para a convergência? O caso do Mercosul, 1990-2014}

\section{RESUMO}

Este artigo responde a dois questionamentos: 1) o processo de integração regional no Mercosul, desde seu lançamento com o Tratado de Assunção (1991), contribuiu para reduzir as desigualdades no ingresso per capita entre os países da região?, 2) ocorreu convergência por meio do comércio intrazona? Para responder a essas perguntas, foi necessário revisar o referencial teórico da integração e buscar explicações que justificassem o processo de integração no Mercosul sob o esquema de união alfandegária e sua vinculação com a convergência. Entende-se a integração como um processo econômico influenciado pelas estruturas produtivas dos países e cujo padrão de comércio responde à designação de verbas da estrutura setorial da produção. Com base no modelo por efeitos fixos, foi observado que as regressões não demonstram existência $\beta$-convergência no PIB per capita do conjunto de países. Além disso, foi estimado um modelo em que foram incluídos o índice de abertura global e o índice de abertura intrarregional. Os resultados não revelam presença $\beta$-convergência condicional para o conjunto de países. Pode-se concluir que o Mercosul não favoreceu o crescimento dos países em termos de PIB per capita.

Palavras-chave: convergência e divergência regional, integração regional, união alfandegária, Mercosul. 


\section{INTRODUCCIÓN}

Acuerdos regionales como los de la Unión Europea (UE), la Asociación de Naciones del Sudeste Asiático (ASEAN) o el Tratado de Libre Comercio de América del Norte (TLCAN) cobran importancia en un mundo pretendidamente globalizado y marcan un precedente en las relaciones económicopolíticas que sostienen los países actualmente. La realidad del modelo de desarrollo dista entonces de la multilateralidad, y son los acuerdos regionales, avalados por el capítulo XXIV de la Organización Mundial del Comercio (OMC), los que caracterizan las relaciones económicas en el ámbito mundial.

El grado de integración, el tipo de países que se integran y el formato de esta dinámica son aspectos determinantes para medir los costos y beneficios del proceso. La teoría ofrece alternativas para la evaluación de si un proceso de integración genera beneficios para el bloque y sus países socios. Una primera medida de costos y beneficios, que asiste desde el inicio mismo del desarrollo de la teoría, deviene de la llamada ambigüedad de Viner (1950), que asume que, al integrarse dos fuerzas contrapuestas, estas operan en dos vías: a) la que va en pro del libre comercio al interno del bloque de integración y b) la que da lugar a un esquema de protección entre el bloque y el resto del mundo. El resultado es un fenómeno de trade-off entre creación de comercio (por el mayor comercio libre al interno del bloque) y el desvío de comercio (por la mayor protección respecto de terceros países al exterior de la zona). De ahí que el efecto sea ambiguo: puede o no haber ganancias de bienestar derivadas de la integración; es decir: "si creación prevalece sobre desvío, habrá ganancias de bienestar".

Otra visión ha sido provista por Meade (1956), quien sostiene que hay beneficios y mayor bienestar derivado de la expansión del comercio y hace hincapié en el beneficio del bloque, mas no en el de los países socios. Recientemente, la discusión de creación y desvío fue complementada por una tercera forma de medición formulada en la década del 2000: habrá mayor bienestar como consecuencia de un proceso de integración, vía unión aduanera, si con el tiempo los ingresos per cápita de los países socios convergen hacia el ingreso del país más desarrollado con mayor bienestar (Venables, 2000). El desarrollo teórico basado en el modelo neoclásico ajustado señala que los acuerdos Norte-Norte y Norte-Sur generan mayores beneficios en comparación con los acuerdos Sur-Sur porque facilitan la convergencia.

Venables (2000) realiza un análisis sistémico de la ventaja comparativa de la unión aduanera y sus efectos sobre el desarrollo industrial en acuerdos simétricos y asimétricos. La formación de una unión aduanera con dos países pobres tiende a producir divergencia en el PIB per cápita, mientras que entre países ricos la integración conduce a la convergencia. Si el país 2 es más pobre que el país 1 , sufre una pérdida del bienestar, al tiempo que este último experimenta un aumento de bienestar.

El Mercado Común del Sur (Mercosur), constituido por el Tratado de Asunción en 1991, agrupa a Argentina, Brasil, Paraguay y Uruguay; también a Venezuela y a Bolivia (la primera en suspensión y la segunda en proceso de adhesión). Este es justamente un modelo de integración Sur-Sur que ha soportado numerosas crisis, la mayor parte de ellas de tipo comercial, debido a temas de ausencia de complementariedad productiva; sin embargo, aún no es claro si la integración ha generado un proceso de convergencia. La integración virtuosa que procuraba el bloque en la primera etapa era poner en marcha un proceso de complementación productiva. Este enfoque permitiría que el Mercosur, en sus primeros cuatro años, pasara de ser una zona de libre comercio (ZLC) a un mercado común. Tal cometido no se logró y llevó a la firma de un acuerdo adicional, el Protocolo de Ouro Preto, de índole comercial, que buscó consagrar el funcionamiento de la ZLC y abandonar la idea del mercado común.

Entre el 2000 y el 2003, el Mercosur se enfrentó a una crisis que estancó las negociaciones, un periodo acompañado de una inactividad en las relaciones comerciales de los países, lo cual llevó a correr la fecha de constitución efectiva de la unión aduanera al 2006. A partir de la Agenda de la Reformulación del Mercosur en la Cumbre de 
Asunción del 2003 y el lanzamiento del Programa de Trabajo 2004-2006, se creó una agenda de trabajo encaminada a la construcción de la unión aduanera; $y$, estando en marcha este procedimiento, Argentina planteó avanzar hacia la unión. La concreción dependería del logro de una serie de objetivos que, en la voz del gobierno, se tradujo como "volver al Tratado de Asunción”. Sin embargo, el Mercosur aún no ha logrado avanzar hacia la liberalización total del comercio intrazona y no ha encaminado un proceso de complementación productiva. Tampoco es claro si se trata de un modelo en el que domine la creación o el desvío de comercio, y tampoco ha sido estudiada la eventual convergencia en el bloque y los países socios. Ambos temas están pendientes de análisis empírico riguroso, y en ese horizonte el presente artículo busca arrojar luz sobre el tema de la convergencia.

Evaluar la evidencia empírica en el marco de la teoría de la unión aduanera, para el proceso de integración del Mercosur, podría ayudar en la interpretación de los resultados obtenidos y en el diseño de un camino favorable para el futuro de la integración en Suramérica. Mercosur es todavía una unión aduanera incompleta y una ZLC parcial, lo que, unido a la característica de integración SurSur, obliga a trabajar con más precisión y dedicación para lograr beneficios de la integración.

\section{MARCO CONCEPTUAL}

El concepto de convergencia fue propuesto por la economía neoclásica del crecimiento y es inherente a los componentes del modelo luego cuestionados por otros modelos de crecimiento endógeno. La relación entre convergencia e integración económica requiere de la adaptación de los conceptos de crecimiento e integración comercial en bloques con preferencia arancelaria, con especial atención a los formatos de unión aduanera y mercado común. Lo anterior, a los efectos de evaluar el aumento del bienestar para el bloque y para cada uno de los países socios.

En términos de bienestar, la convergencia intrabloque es un requisito para el éxito de la integración. Es claro que el logro de convergencia requiere el funcionamiento de una unión aduanera completa (o de un mercado común completo) y la existencia de coordinación macroeconómica, a lo que se suma la ausencia de políticas de países individuales y no planificadas en bloque que impliquen incentivos o subsidios a la inversión, la producción, el consumo y la exportación.

El análisis teórico y la evidencia de los procesos de integración económica coinciden en la búsqueda de mayor bienestar para los países socios de un área preferencial de comercio (APC). La visión teórica y de la política económica de la integración concibe que el desarrollo económico, más allá del crecimiento del PIB de los países, es la base de ese bienestar. Los países que buscan asociarse tras barreras comerciales y con preferencias intrabloque apuestan a la vía del comercio internacional para dinamizar sus economías. Se espera que la formación de una APC provea el blindaje necesario para abordar una reorganización de la asignación de factores, así como la búsqueda de un uso pleno de las ventajas comparativas y el desarrollo de ventajas competitivas.

La medición del bienestar ha comenzado por el enfoque de Viner (1950) y Lipsey (1960), sobre la "creación y desvío de comercio", y el de Meade (1956), de "expansión de comercio", unidos a la advertencia del análisis teórico de Venables (2000) sobre los riesgos de las alianzas Sur-Sur. No obstante, con independencia de estas miradas, se trata de encontrar un mecanismo práctico que permita observar los progresos de una APC en términos de bienestar en cada uno de los países miembros. Un indicador posible y medible es el de convergencia del PIB per cápita, para lo cual es necesario considerar como variable independiente el comercio exterior, sea a través de la intensidad comercial (trade intensity) o el peso del comercio intraindustrial en el intercambio intrazona. La convergencia hacia el "mejor" PIB per cápita de los socios reflejaría un progreso en términos de bienestar, resultado del incremento de la equidad entre los países miembros de la unión aduanera. 
La teoría del crecimiento, en su versión del modelo neoclásico, intenta estudiar el papel del capital físico como motor fundamental de la economía. Su enfoque se centra principalmente en los rendimientos decrecientes y en cada factor (capital, trabajo). La medición utilizada para esta clase de estudios empíricos se hace mediante la $\beta$-convergencia. Por su parte, la teoría de crecimiento endógeno considera que el nivel de renta per cápita puede crecer sin límites, dependiendo del nivel de progreso técnico. Asimismo, enfatiza en el papel del conocimiento, destacando que este puede ser transferido con la liberalización de los mercados comerciales de bienes.

\section{REVISIÓN DE LITERATURA}

A pesar de la acumulación de experiencias en la formación de procesos de integración en América Latina (Asociación Latinoamericana de Libre Comercio [ALALC], 1960-1980; Asociación Latinoamericana de Integración [ALADI], 1980-actualmente; Comunidad Andina de Naciones [CAN], 1969-actualmente, y Mercosur, 1991-actualmente) $)^{1}$, no abundan los estudios de convergencia para estos bloques de comercio preferencial.

Karras (1997) analiza tres acuerdos de integración regional entre 1960 y 1990. Para la Unión Europea (UE), examina 15 países, algunos de la ASEAN y otros de ALALC, y concluye dos resultados principales: a) en la UE los países experimentan un proceso de convergencia y b) en la ALALC los países tienden hacia una convergencia más débil, mientras que en el ASEAN hay ausencia de convergencia. El autor concluye que la integración no garantiza la convergencia en el PIB per cápita; sin embargo, deja claro que los efectos de la integración sí pueden estar relacionados con el tipo de socio. Lo anterior significa que, según las características

$1 \quad$ La ALALC abarcó los países de América del Sur y México; la ALADI opera a través mecanismos de preferencia arancelaria regional: acuerdos de alcance regional (participan todos los países miembros) y acuerdos de alcance parcial (con la participación de dos o más países); la CAN se formó con Bolivia, Chile, Colombia, Ecuador, Perú y Venezuela, en tanto Chile y Venezuela salieron de la zona posteriormente. de los países frente a sus socios, se puede predecir el resultado que se obtendrá con la integración de los países (Norte-Norte, Sur-Sur, Norte-Sur) (Schiff y Winters, 2003, pp. 128-129).

El trabajo realizado por Ramón (2003) se centra en comprobar convergencia en las provincias argentinas y brasileñas en las décadas de 1970 a 1990. Analiza el impacto de la inestabilidad política y macroeconómica sobre el proceso de convergencia entre Argentina y Brasil. Los resultados muestran que las disparidades entre estos países no se han reducido considerablemente en las últimas décadas. El autor resalta ausencia de políticas regionales y una necesidad de la construcción de entes supranacionales. Desde la perspectiva de la convergencia sigma, se observa que la dispersión de los ingresos per cápita se reduce entre 1991 y 1995, es decir, en este periodo la desigualdad de los ingresos fue menor, aunque crece en los años siguientes. De la función de densidad Kernel se concluye que el PIB per cápita tanto entre países como regiones aumenta después de 1995. El trabajo encuentra que "ha existido una desigualdad muy marcada entre las regiones del bloque y, por lo tanto, se han conformado regiones de 'centro' y 'periferia'” (Arias, 2008, p. 75).

Blyde (2007) toma como referencia 15 regiones del Mercosur con el fin de contrastar tres medias de desigualdad: a) convergencia sigma, b) coeficiente de Gini y c) índice de Theil. Los resultados muestran que a partir del 2000 la desigualdad aumentó en las regiones analizadas. El autor deduce que, a pesar del aumento de las disparidades, la integración habría tenido un papel importante en la reducción de la desigualdad regional, debido a la movilización de individuos hacia regiones con mejores oportunidades. Lo anterior ha permitido la formación de clubes de regiones ricas y clubes de ingresos bajos e ingresos medios-bajos.

Cresta (2008) estudia los niveles de crecimiento y desarrollo vinculados con el proceso de integración regional en el Mercosur, entre 1985 y 2003. Los resultados indican que no hay evidencia de un proceso de convergencia absoluta en el PIB per cápita. En el panel de datos, por efectos fijos 
que permiten examinar la hipótesis de convergencia condicional, los resultados a partir de 1995 evidencian que la velocidad de convergencia cayó en las regiones en las que se encontraron retardadores de convergencia (noreste de Brasil, norte de Argentina y Paraguay) y aumentó donde se encontraron efectos impulsores (sudeste, sur y centro-oeste de Brasil, resto de Argentina y Uruguay).

El trabajo de Brida et al. (2012) observa evidencia favorable de la convergencia por clubes de desempeño en las provincias y estados en el Mercosur. Así, se evidencia la conformación de regiones ricas y pobres entre 1961 y 2005 . Las regiones mejor posicionadas en Brasil confirman un proceso de convergencia interregional con las regiones más ricas de Argentina y Uruguay. Respecto a Paraguay, sus regiones guardan correspondencia con las regiones del nordeste de Brasil y las regiones argentinas históricamente más rezagadas, donde persiste la divergencia. Las bondades de la integración pueden advertirse pero no asegurarse a lo largo del proceso. "La mayor integración, por ejemplo, puede conducir a un desarrollo desigual dentro de un país y la renta nacional dejará de captar este tipo de divergencia" (Blyde, 2007, p. 791).

\section{INTEGRACIÓN COMERCIAL DEL MERCOSUR}

La idea de Mercosur pivotó en torno al crecimiento económico y al aumento del bienestar. El aumento en el flujo de comercio intrazona sería el motor que impulsaría la asignación de recursos, y esto se alinearía con las ventajas comparadas. Sin embargo, la especialización, la complementación productiva, las economías de escala y el incremento en la productividad no responderían automáticamente a la liberalización del comercio. Así, la expansión del comercio al interno del bloque simplemente aseguraría un aumento en los flujos y una asignación de divisas en función de los saldos bilaterales, pero poco se podría decir de ganancias y pérdidas del comercio para el bloque e incluso para los Estados Parte, vía la "expansión" á la Meade, o el trade-off entre "creación y desvío" de comercio á la Viner.
La figura 1 muestra la expansión del volumen de comercio intrazona ${ }^{2}$, al tiempo que señala el bajo nivel de "intensidad de comercio" ${ }^{\text {. Mientras el }}$ comercio del Mercosur al interior creció once veces entre 1991 y 2014, la "intensidad del comercio" se mantuvo un poco por encima de los niveles del coeficiente en tiempos del lanzamiento del Tratado de Asunción. En aquellos años la "intensidad" fue de $6 \%$ y para la década de los 2000 se mantendría entre $7 \%$ y $8 \%$. En la época de oro del Mercosur, 1995-1998, el coeficiente se movió entre $10 \%$ y $11 \%$, muy lejos de las cifras que mostraron la Unión Europea, el Tratado de Libre Comercio de América del Norte (NAFTA) y la Asociación de Naciones del Sudeste Asiático (ASEAN).

Los datos muestran el crecimiento del comercio intrazona desde la firma del Tratado de Asunción, que representó, sin duda, un logro de la integración. De USD $\$ 4000$ millones de intercambio en 1990 se pasaron a casi USD $\$ 53.000$ millones en el 2011. Sin embargo, habría que observar el patrón de comercio, algo importante no solo por el signo del saldo del intercambio, sino porque el balance comercial por sectores es un buen indicador de ventajas comparativas reveladas (VCR), lo que a su vez puede reflejar el patrón de producción doméstico.

La tabla 1 muestra los saldos de la balanza comercial entre Argentina y Brasil, que representan en conjunto el $96 \%$ del comercio del Mercosur. Al año de iniciado este proceso de integración, las exportaciones de manufacturas de origen industrial (MOI) registraban un déficit de USD \$2230,7 millones, que subió a USD \$7412,1 millones en el 2011. Sin embargo, en el 2014 se vuelve a cifras de 1998. Tanto en 1992 como en el 2011 todos los sectores industriales registraban déficit para Argentina. El volumen del comercio, medido en USD\$ corrientes, creció once veces entre 1991 y 2014 ,

2 Medido como el cociente entre el comercio intrazona, que resulta de la suma de las exportaciones de cada uno de los países miembros a los restantes de la región.

3 Representado por el coeficiente de intensidad de comercio, en porcentaje, que resulta del cociente entre la suma de exportaciones e importaciones de cada uno de los países al resto de la región y la suma de las exportaciones e importaciones totales de cada uno de los cuatro países de la región a todos los destinos, incluyendo el propio Mercosur. 
Figura 1.

Interrelación del comercio de los países miembros del Mercosur, 1990-2014

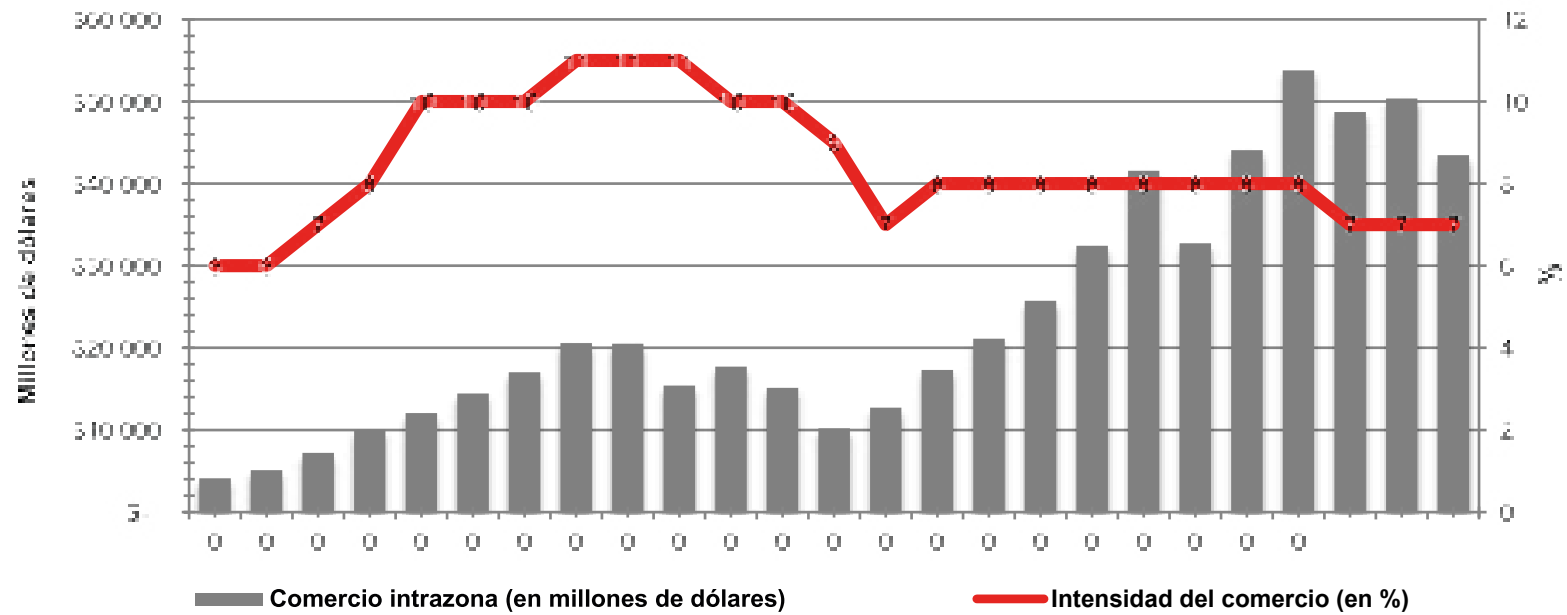

Fuente: elaboración propia con base en CEI.

con importaciones argentinas desde Brasil aumentando 14,2 veces y las exportaciones de Argentina a Brasil creciendo 11,6 veces, entre ambos años.

El patrón de comercio de Argentina, medido a través del saldo del comercio bilateral, muestra un superávit creciente en materias primas, en combustible y en manufacturas de origen agropecuario (MOA), excepto calzado. Justamente este sector productivo, que tenía un comercio equilibrado con Brasil en la década de 1990, sesgó su saldo hacia negativo en el 2014, cuando el país experimentó un saldo favorable de USD \$118 millones.

Tabla 1.

Saldo de balanza comercial Argentina-Brasil por segmento (en millones de USD)

\begin{tabular}{|c|c|c|c|c|c|c|}
\hline Producto/año & 1992 & 1995 & 1998 & $\mathbf{2 0 0 5}$ & $\mathbf{2 0 1 1}$ & $\mathbf{2 0 1 4}$ \\
\hline Productos primarios & 439,7 & 1385,50 & 1644,10 & 662,3 & 1767,90 & 1646,00 \\
\hline Agropecuario, silvicultura y pesca & 583,9 & 1537,10 & 1815,10 & 1035,70 & 3019,90 & 1885,00 \\
\hline Minería-extracción & $-144,2$ & $-151,6$ & $-171,0$ & $-373,4$ & $-1252,00$ & $-239,00$ \\
\hline Manufacturas de origen agropecuario & 19,0 & 226,5 & 276,7 & 40,5 & $-94,4$ & $-289,6$ \\
\hline Alimentos & $-27,4$ & 123,9 & 276,1 & 112,6 & 143,7 & $-158,0$ \\
\hline Madera-muebles & $-6,2$ & $-0,3$ & $-31,9$ & 22,8 & 9,4 & $-9,6$ \\
\hline Cuero y manufacturas & 54,2 & 74,7 & 74,7 & 27,1 & $-3,4$ & $-4,0$ \\
\hline Calzado & $-1,6$ & 28,2 & $-42,4$ & -122 & $-244,1$ & $-118,0$ \\
\hline Combustibles y aceites minerales & 96,1 & 689,8 & 631,7 & 819,6 & 1286,50 & 540,00 \\
\hline Manufacturas de origen industrial & $-2230,70$ & $-1013,50$ & $-1644,00$ & $-5370,20$ & $-7412,10$ & $-1714,00$ \\
\hline Textil e indumentaria & $-111,2$ & 87,9 & $-29,4$ & $-311,5$ & $-236,3$ & $-130,0$ \\
\hline Papel, manufacturas e industria gráfica & $-136,6$ & $-112,6$ & $-174,2$ & $-223,6$ & $-373,6$ & $-266,0$ \\
\hline Química, caucho, plástico y sus productos & $-331,1$ & $-530,1$ & $-528,2$ & $-496,0$ & $-1224,20$ & $-396,00$ \\
\hline Siderurgia-metalurgia & $-109,9$ & $-170,2$ & $-1043,70$ & $-679,0$ & $-1221,90$ & $-594,00$ \\
\hline Maquinaria, electrodomésticos y otros & $-473,7$ & $-296,7$ & $-198,1$ & $-1780,40$ & $-2789,80$ & $-1332,00$ \\
\hline Automotores y autopartes & $-695,6$ & 311,9 & 452,9 & $-1703,60$ & $-1073,50$ & 1130,00 \\
\hline Otros MOI & $-372,6$ & $-303,9$ & $-123,2$ & $-176,1$ & $-492,6$ & $-126,0$ \\
\hline Total general & $-1675,80$ & 1288,30 & 908,5 & $-3847,80$ & $-4452,10$ & 182,40 \\
\hline
\end{tabular}

Fuente: elaboración propia con base en INTAL. 
Desde un punto de vista vineriano, Argentina ha estado exportando a Brasil volúmenes crecientes de productos commodities a precios internacionales, pero ha estado comprando cantidades crecientes de bienes industriales fuertemente protegidos en el Mercosur, con tarifas que para bienes de consumo, en el 2011 , se ubicaron en aranceles del $20 \%$ al $35 \%$. Bajo este movimiento, no habría aumentos en el bienestar de Argentina, aunque sí podría tenerlos en Brasil. Tampoco puede argumentarse á la Meade que la notable expansión del comercio haya generado bienestar, porque no se sabe ahora cuáles fueron los costos de la protección, vis a vis, en el mercado mundial. El aumento del comercio global del Mercosur, mostrado en la figura 1, tampoco asegura beneficios de aumento de bienestar por convergencia en el PIB per cápita, porque la "intensidad" es baja y, por ende, la interrelación por el canal del comercio deberá ser baja.

\section{METODOLOGÍA: ¿CONVERGENCIA EN EL MERCOSUR?; UN MODELO DATA PANEL}

\section{Especificación del modelo}

Se mide la $\beta$-convergencia absoluta y $\beta$-convergencia condicional recogiendo los criterios expuestos por Sala-i-Martin (2000), sobre la base del modelo de crecimiento neoclásico y su extensión al modelo de crecimiento endógeno, con el fin de establecer si el índice de apertura intrarregional y el índice de apertura global, como variables explicativas, influyeron en la convergencia o divergencia de los países del Mercosur. La convergencia beta se calcula a partir de la siguiente ecuación general:

$$
\left(\operatorname{Iny}_{t}-\operatorname{In} y_{t-1}\right)=\beta_{0}+\beta_{1} \operatorname{Iny}_{t-1}+\varepsilon_{t}
$$

\section{Donde:}

- $\quad\left(\ln y_{t}-\ln y_{t-1}\right)$ es la primera diferencia del PIB per cápita, es decir, la variación porcentual de un periodo a otro (indicador de crecimiento).

- $\quad \beta_{1}$ mide la velocidad de la convergencia; en la medida en que beta sea mayor, la velocidad de convergencia será directamente proporcional.
- $\quad \operatorname{lny}_{t-1}$ es el logaritmo natural en el PIB per cápita; mide la elasticidad de este ante cambios en la velocidad de convergencia en el periodo inmediatamente anterior.

- $\quad t$ representa el periodo.

El parámetro $\beta_{1}$ indica si hubo convergencia o no en la región. Si el parámetro es negativo, indicará que los países con mayor PIB per cápita crecieron menos que, proporcionalmente, los países con menor PIB per cápita. Lo anterior demostrará que la brecha entre países, a niveles de producto per cápita, se está cerrando mediante el proceso de convergencia. Por otro lado, si $\beta_{1}$ resulta positivo, refleja que los países tienden a divergir en el largo plazo.

Para estudiar el impacto en la "convergencia" del comercio intrabloque y total, dado que la integración comercial —en especial a partir de Ouro Preto- se transformó en el parámetro con el que se mide usualmente el progreso de la integración Mercosur, se incorporan al análisis y a la medición data panel variables sobre el grado de apertura comercial. La ecuación toma la siguiente forma:

$\left(\right.$ Iny $\left._{t}-\operatorname{Iny} y_{t-1}\right)=\beta_{0}+\beta_{1}$ Iny $_{t-1}+\varnothing z_{t}+\varepsilon_{t}$

Donde el parámetro $Z_{t}$ indica la inclusión de la variable de apertura económica, que está determinada por las exportaciones e importaciones (reunidas en el índice de apertura global e intrarregional $)^{4}$, para la serie de tiempo 1990-2010. El cambio en los logaritmos de la variable de interés ( $\Delta$ Iny) es igual a la tasa de crecimiento de la variable, mientras que $\operatorname{lny}_{t-1}$ es el nivel de producto per cápita, en el periodo $t-1$, y $\varepsilon_{t}$ es un error aleatorio en el tiempo.

Se asume que, dentro de los parámetros de los modelos de crecimiento neoclásico, crecimiento endógeno ${ }^{5}$ y del comercio, un mayor nivel de apertura conduce a un mayor ritmo de crecimiento. Dado que una característica constitutiva de las uniones

\footnotetext{
$4 \quad$ El índice de apertura está determinado por la suma de las exportaciones e importaciones sobre el PIB.

5 La nueva teoría del crecimiento endógeno considera que la apertura comercial, entendida como el volumen de bienes y servicios que se transan de una economía a otra, y viceversa, genera un mayor dinamismo económico y, por ende, crecimiento.
} 
aduaneras implica apertura intrazona sin restricciones, la formación de un bloque de integración debería contribuir al crecimiento económico de los países miembros, inclusive cuando puedan suscitarse crisis comerciales.

El caso por examinar es si, dado el mayor nivel de comercio intrazona, el crecimiento por el comercio puede facilitar la "convergencia" de las economías del bloque. La literatura no es concluyente. Algunos trabajos plantean que cuanto mayor sea la apertura de los mercados, mayor será el nivel de difusión del conocimiento al cual están expuestos los países. Se esperaría que con la integración los países aumenten el stock global de conocimiento, por lo que este se acumulará más rápido respecto a los países que no forman parte de ningún acuerdo comercial. El trabajo realizado por Sachs y Warner (1995, citado en Díaz, 2003) incluye la variable cuantitativa de apertura económica en las regresiones de "convergencia".

Los resultados obtenidos por estos investigadores muestran que la liberalización comercial ${ }^{6}$ y el crecimiento económico tienen una relación positiva. En el caso de modelos de integración económica, de la relación comercio-crecimiento - y, quizás, la relación comercio-"convergencia"- podría esperarse algún efecto positivo en la causación, sobre todo porque la teoría de la integración se fundamenta en la teoría del libre comercio (desmantelamiento de la protección), y a esta se le estudia como rama de la teoría de tarifas (teoría positiva del comercio) y como rama de la teoría del bienestar en el comercio (ganancias y pérdidas) (Corden, 1965).

Se realizaron regresiones para el análisis de la $\beta$-convergencia y $\beta$-convergencia condicional. Esta última se divide en dos momentos: a) la estimación en conjunto y b) su determinación por los modelos por país. El panel de datos se construye sobre la base de información de la Comisión Económica para América Latina (Cepal), de donde se obtuvieron los datos del ingreso per cápita

6 Díaz (2003) mide la convergencia condicional para las regiones mexicanas e incluye en su modelo el parámetro $Z_{t}$, que recoge una serie de variables estructurales, institucionales y de política económica (índice de apertura). (a precios constantes del 2010). Los datos correspondientes al comercio -discriminados en los índices de apertura global e intrarregional ${ }^{7}-\mathrm{y}$ las exportaciones e importaciones, para la construcción de los índices, fueron tomados de las estadísticas de la ALADI. La serie de tiempo está delimitada por el periodo 1990-2014, con la información para los cuatro Estados miembro del Mercosur.

\section{RESULTADOS Y ANÁLISIS}

\section{Elección del modelo: efectos fijos o aleatorios}

MODELO POR EFECTOS FIJOS ${ }^{8}$. De acuerdo con los resultados obtenidos ${ }^{9}$, el modelo por efectos fijos es estadísticamente significativo a un nivel de confianza del $95 \%$; es decir, los parámetros son significativos y confiables estadísticamente (hipótesis alterna $\mathrm{H}_{1} \neq 0$ ). Los efectos fijos solo utilizan variación "within" de los datos, lo cual significa que la variabilidad está en torno a la media individual, o sea, en el tiempo para un individuo dado (tabla 2).

En cuanto a la significancia del tipo de ajuste del modelo, se utiliza la prueba $F$, con las siguientes hipótesis: a) $H_{0}$ : las variables independientes no

$7 \quad$ Con la información de las exportaciones e importaciones globales e intrarregionales tomadas de las estadísticas de la ALADI y el PIB, a precios constantes del 2000, en millones USD\$; a partir de las estadísticas de la Cepal se construyeron los índices de apertura global e intrarregional utilizados en el modelo econométrico. Índice de apertura global $=$ exportaciones globales $($ país $)+$ importaciones globales / PIB constante a precios del 2000. Índice de apertura intrarregional $=$ exportaciones intrarregionales + importaciones intrarregionales / PIB constante a precios del 2000. Las cifras son tomadas de la base de datos de la ALADI en millones de dólares.

8 Los modelos por efectos fijos consideran que las variables explicativas del modelo afectan por igual a las unidades de corte transversal. Esto significa que cada país tiene sus propias características individuales, que pueden o no influir en las variables predictivas, que en este caso estarían vinculadas al efecto del comercio intrarregional y global sobre el crecimiento del PIB per cápita de cada país.

9 En el texto referido expresamente a las mediciones econométricas se explican los estimadores importantes y relevantes para la modelización data panel, es decir, aquellos que se relacionan con la veracidad del modelo (p. e., si tiene pendiente e intercepto), lo que habla de si el modelo es estadísticamente significativo. 
Tabla 2.

Estimación del modelo por efectos fijos ( $\beta$-convergencia)

\begin{tabular}{|c|c|}
\hline Coeficientes estadísticos & $\begin{array}{c}\text { Convergencia en el PIB per } \\
\text { cápita }\end{array}$ \\
\hline$\beta$ & 0,070532 \\
\hline Estadístico $t$ & 2,51 \\
\hline Probabilidad (valor-p) & 0,014 \\
\hline Estadístico $F$ & 6,32 \\
\hline Número de observaciones & 96 \\
\hline
\end{tabular}

Fuente: elaboración propia con base en las estimaciones realizadas en STATA 11.

explican la variable endógena; b) $H_{1}$ : las variables independientes explican la variable endógena. Se concluye entonces que las variables entre sí explican la variable independiente. El $F$ calculado $(6,30)$ es mayor al $F$ de la tabla $(3,47)$, por lo cual se rechaza la hipótesis nula. Se puede observar a su vez que la correlación de los ajustes $(-0,9449)$ es alta, lo que refleja una relación alta con los parámetros de forma inversa. Por su parte, el coeficiente de la medida de desigualdad resulta ser positivo $(0,070532)$, lo cual indica la no existencia de $\beta$-convergencia.

En términos económicos, los resultados indican que en el Mercosur los países no han experimentado un crecimiento económico que los lleve en el largo plazo a alcanzar un nivel de renta per cápita similar. Las disparidades entre los países del bloque tienen un peso importante en el desarrollo y crecimiento de sus regiones. Conviene señalar que el crecimiento económico guarda una relación positiva con la estabilidad política y económica, el mantenimiento de la ley y el orden, la inversión pública (educación, salud, infraestructura) y la inversión en capital físico y maquinaria (Sala-i-Martin, 2000, p. 216).

MODELO POR EFECTOS ALEATORIOS ${ }^{10}$. El modelo es significativo estadísticamente en su conjunto,

10 Los modelos por efectos aleatorios parten de este principio: "Los efectos individuales no son independientes entre sí, sino que están distribuidos aleatoriamente alrededor de un valor dado" (Mayorga y Muñoz, 2000, p. 8). La especificación econométrica de este modelo es la misma de los efectos fijos, con la diferencia de que, en lugar de ser un valor fijo para cada país y un valor constante a lo largo del tiempo, es una variable aleatoria con un valor la probabilidad $\mathrm{Chi}^{2}$ es menor al 0,05 $(0,00)$, lo que significa que las variables independientes explican la variable endógena. Esto también significa que los coeficientes son diferentes a cero $\left(\mathrm{H}_{1} \neq 0\right)$ (tabla 3$)$.

Tabla 3

Estimación del modelo por efectos aleatorios $(\beta$-convergencia)

\begin{tabular}{|c|c|}
\hline $\begin{array}{c}\text { Coeficientes } \\
\text { estadísticos }\end{array}$ & $\begin{array}{c}\text { Convergencia en el PIB per } \\
\text { cápita }\end{array}$ \\
\hline$\beta$ & 0,01378 \\
\hline Prueba normal $z$ & 1,67 \\
\hline Probabilidad (valor-p) & 0,096 \\
\hline Chi $^{2}$ & 2,78 \\
\hline $\begin{array}{c}\text { Número de } \\
\text { observaciones }\end{array}$ & 96 \\
\hline
\end{tabular}

Fuente: elaboración propia con base en las estimaciones realizadas en STATA 11.

La prueba de la normal $Z$ muestra que los coeficientes son estadísticamente significativos a un nivel de confianza del $5 \%$. El coeficiente del logaritmo natural del PIB per cápita es estadísticamente significativo al nivel de confianza del $95 \%$, es positivo $(0,0137888) \mathrm{y}$, al igual que en el modelo por efectos fijos, no se encuentra evidencia de $\beta$-convergencia. En cuanto a los coeficientes de bondad de ajuste (within, between, overall), estos revelan que el modelo por efectos aleatorios tiene la misma ponderación "between" que en el modelo por efectos fijos. Lo anterior significa que tanto el modelo por efectos fijos como el modelo por efectos aleatorios asumen la misma variabilidad entre los países del bloque.

Ahora bien, hecha la prueba de Hausman ${ }^{11}$, se determina un $\mathrm{Chi}^{2}$ de 4,48 y una $\mathrm{Prob}>\mathrm{Chi}^{2}$ igual a 0,0343 (menor a 0,05), lo cual lleva a rechazar

medio $v_{i}$ y una varianza $\operatorname{Var}\left(v_{i}\right) \neq 0$ (Montero, 2011, p. 2). Se dice entonces que las diferencias entre las unidades de corte transversal (países) tienen influencia en la variable dependiente (PIB per cápita). En consecuencia, el modelo por efectos aleatorios es más dinámico.

11 "La prueba de Hausman se aplica para comprar de manera formal los estimadores mínimos cuadrados ordinarios (MCO) y los mínimos cuadrados ponderados (MCP) con el fin de ver si difieren en algo más que los errores muestrales" (Wooldridge, 2001, p. 271). Básicamente esta prueba muestra si los errores se correlacionan con los regresores. 
la hipótesis nula, es decir, se debe seleccionar el estimador para efectos fijos:

Hipótesis nula: $\mathrm{H}_{0}$ : efectos aleatorios

Hipótesis alterna: $\mathrm{H}_{1}$ : efectos fijos

El modelo por efectos fijos considera que cada país tiene sus propias características individuales, las cuales pueden o no influir en las variables predictivas (en este caso estarían vinculadas al efecto del comercio intrazona y global sobre el crecimiento del PIB per cápita de cada país).

MODELO POR EFECTOS FIJOS: $\beta$-CONVERGENCIA $Y \beta$-CONVERGENCIA CONDICIONAL. Dados los resultados obtenidos de las pruebas realizadas, el modelo por efectos fijos es el que mejor se ajusta a los datos presentados para este trabajo. Las pruebas se realizaron con 24 observaciones por país (96 observaciones en total, al considerar un retardo en el periodo $t-1$ ), para la serie de tiempo comprendida entre 1990 y 2014. Los parámetros resultaron ser estadísticamente significativos a un nivel de confianza del $95 \%$. La probabilidad de 0,013 de la $F$ da muestra de un modelo válido en conjunto. Esto significa que las variables independientes explican la variable endógena. El modelo, a partir de los ejercicios realizados, arrojó los siguientes resultados:

$$
\begin{gathered}
\operatorname{In}\left(P I B P-P I B P_{t-1}\right)= \\
-0,6028+0,70532 \operatorname{InPIBP}_{t-1}+\varepsilon_{t-1} \\
\text { ee }(0,280634)(0,24841) \\
\text { pb }(0,017)(0,014)
\end{gathered}
$$

Dado que $\beta_{1}$ es positivo $(0,70532)$, se puede concluir que no hay evidencia de $\beta$-convergencia para el conjunto de países. Esto significa que los países con menor PIB per cápita al interior del bloque (Brasil y Paraguay) en el largo plazo no alcanzaron tasas de crecimiento que les permitieran converger en el largo plazo hacia los niveles de PIB per cápita más altos (Argentina y Uruguay). El siguiente paso fue estimar un modelo que incluyera el índice de apertura intrarregional (intrazona) y el índice de apertura global (con el mundo) ${ }^{12}$,

12 Asume todos los países, incluyendo Mercosur. representados a través del parámetro $Z_{t}$, tal como se muestra en la ecuación [1]:

$\left(\operatorname{Iny}_{t}-\ln y_{t-1}\right)=\beta_{0}+\beta_{1} \ln y_{t-1}+\varnothing z_{t}+\varepsilon_{t}$

El objetivo fue comprobar si hay evidencia de $\beta$-convergencia condicional ${ }^{13}$, es decir, determinar si la tasa de crecimiento del ingreso per cápita está inversamente relacionada con la distancia que los separa de su propio estado estacionario. La estimación y explicación del modelo se hace bajo el modelo por efectos fijos, como en el ejercicio anterior (tabla 4).

Tabla 4.

Estimación del modelo por efectos fijos ( $\beta$-convergencia condicional)

\begin{tabular}{|c|c|}
\hline Coeficientes estadísticos & $\begin{array}{c}\text { Convergencia en el PIB per } \\
\text { cápita }\end{array}$ \\
\hline$\beta$ & $-0,1830583$ \\
\hline Estadístico $t$ & $-4,15$ \\
\hline Probabilidad (valor-p) & 0,000 \\
\hline Estadístico $F$ & 0,000 \\
\hline Número de observaciones & 96 \\
\hline
\end{tabular}

Fuente: elaboración propia con base en las estimaciones realizadas en STATA 11.

En su conjunto, los parámetros son significativos a un nivel de confianza del $95 \%$. La significancia del tipo de ajuste, medida por la prueba $F$, muestra que las variables independientes explican la variable endógena (los parámetros son diferentes de cero). Se puede apreciar que la correlación en el ajuste es alta y negativa $(-0,9777)$, lo que significa que las variables independientes explican a la variable dependiente en un $97 \%$ de forma inversa. El panel data arrojó los siguientes resultados:

$$
\begin{aligned}
& \operatorname{In}\left(P I B P-P I B P_{t-1}\right)=1,67-0,18 \operatorname{InPIBP} P_{t-1} \\
& +0,69 \operatorname{InI} A G-0,32 \operatorname{InI} I R+\varepsilon_{t} \\
& \text { ee }(0,397) \quad(0,044) \quad(0,018) \quad(0,017) \\
& p b(0,000)(0,000) \quad(0,000) \quad(0,071)
\end{aligned}
$$

13 Si $\beta$ es negativo y estadísticamente significativo, se cumple con la hipótesis de la convergencia condicional. Para comparar economías con diferencias estructurales, es necesario incluir variables que ayuden a explicar los cambios en el nivel del PIB per cápita, lo cual se hace en este artículo con la inclusión de las variables comerciales (índice de apertura global e índice de apertura intrarregional). 
Como el parámetro $\beta_{1}(0,18)$ es negativo, se confirma la convergencia en los niveles de ingreso per cápita para el conjunto de países, condicionada a las variaciones de los índices de apertura global e interregional. El tipo de ajuste por efectos fijos asume que las variables explicativas afectan por igual a cada uno de los países. Esto significa que la variación en el PIB per cápita incorpora igual intercepto y sensibilidad para cada país del Mercosur, ante cambios en los índices de apertura global e intrarregional. La relación del índice de apertura global y el crecimiento del PIB per cápita del bloque es positiva.

Asimismo, ante una variación del PIB per cápita, el índice de apertura global aumenta, mientras que el índice de apertura intrarregional es inversamente proporcional. En efecto, por un $1 \%$ que aumente el comercio intrarregional el PIB per cápita, del conjunto de países disminuye en un $0,32 \%$, ceteris paribus. Mientras que por $1 \%$ que aumente el comercio global, el PIB per cápita del conjunto de países se incrementa en 0,69\%. Este resultado es relevante y refleja los hechos reales de las economías del bloque: el comercio intrazona no resulta positivo para el bloque, mientras que sí lo sería el comercio global.

Los hechos estilizados en las ecuaciones ponen en evidencia que las crisis o conflictos comerciales no resultan triviales en términos de beneficios de la integración. Lo que está detrás de los conflictos - de ahí el ajuste negativo para el comercio intrabloque- es la falta de complementación en el patrón productivo ${ }^{14}$.

\section{Estimación del modelo por países}

La tabla 5 ofrece el resumen de las estimaciones realizadas para determinar si hay evidencia de

14 El caso paradigmático es el de la industria automotriz, que, dada la imposición del Régimen Automotor Brasileño en 1995, rompió unilateralmente lo acordado en Ouro Preto (ver Decisión CMC 29/94), a lo que se sumó el Régimen Automotor del Nordeste (diciembre 1996) y los sucesivos programas de incentivos y subsidios para atraer inversiones, sea por la Unión o por los Estados, todo lo cual afectó el patrón productivo $\mathrm{y}$, por tanto, el comercio. Todas las crisis "automotrices" bilaterales, hasta la actualidad, vienen de aquellas medidas. convergencia condicional para cada país miembro del Mercosur. El modelo por país, en su conjunto, es estadísticamente significativo.

El modelo para Argentina es significativo; sin embargo, la probabilidad del índice intrarregional no explica en un alto porcentaje los cambios en el PIB per cápita. El parámetro $\beta_{1}(0,2508)$ es negativo y estadísticamente significativo, resultado que indicaría que el Mercosur, para el caso de Argentina, da indicios para pensar que la integración ha sido factor de "convergencia". Argentina es el país Mercosur con más alto PIB per cápita; por ende, la convergencia no sería un beneficio per se, dado que debería ajustar hacia abajo o crecer menos que sus vecinos socios o mantenerse sin crecimiento, para facilitar la convergencia del bloque. En términos simples, la convergencia para Argentina no sería necesariamente un éxito, aunque podría crecer y "tolerar" convergencia. En este caso habría eventualmente beneficios de tener un mercado regional más importante, de mayor tamaño, lo que podría facilitar el comercio virtuoso; pero esto requiere previamente acuerdos de complementación.

En los hechos, resulta que la ecuación data pane/ para Argentina estaría ratificando la intuición; es decir, Mercosur debería tener ajustes que permitan al país obtener beneficios claros y netos, no solo en términos de saldo de comercio, sino también en asignación de recursos, escala, especialización y productividad, lo que no parece estar sucediendo.

Ecuación 1: Argentina $\beta$-convergencia condicional: In $\left(P I B P-P I B P_{t-1}\right)=2,4109-0,2508 \operatorname{InPIBP} P_{t-1}$ $+0,1172 \ln I A G-0,0384 \ln I A I R+\varepsilon_{t}$

$$
\begin{array}{llll}
\text { ee }(0,952) & (0,993) & (0,571) & (0,040) \\
p b(0,011) & (0,012) & (0,040) & (0,347)
\end{array}
$$

El análisis se ratifica a partir de los resultados obtenidos en las regresiones, que para Argentina muestran una relación positiva entre el índice de apertura global y el crecimiento del país, mientras que su relación con el índice de apertura intrarregional es negativa. Esto indica que por un $1 \%$ que aumenta el índice de apertura global, la variación en el crecimiento del país es del $0,11 \%$. Por otro lado, por un $1 \%$ que aumenta el índice de apertura 
Tabla 5.

Resultados de estimación del modelo por países (Mercosur)

\begin{tabular}{|c|c|c|}
\hline País & Coeficientes estadísticos & Convergencia en el PIB per cápita \\
\hline \multirow{5}{*}{ Argentina } & $\beta$ & $-0,2508$ \\
\hline & Prueba normal $Z$ & $-2,53$ \\
\hline & Probabilidad (valor-p) & 0,012 \\
\hline & $\mathrm{Chi}^{2}$ & $-0,4455$ \\
\hline & Número de observaciones & 24 \\
\hline \multirow{5}{*}{ Brasil } & $\beta$ & $-0,5116$ \\
\hline & Prueba normal $Z$ & $-6,20$ \\
\hline & Probabilidad (valor-p) & 0,000 \\
\hline & $\mathrm{Chi}^{2}$ & $-0,6733$ \\
\hline & Número de observaciones & 24 \\
\hline \multirow{5}{*}{ Paraguay } & $\beta$ & $-0,2523$ \\
\hline & Prueba normal $Z$ & $-2,12$ \\
\hline & Probabilidad (valor-p) & 0,034 \\
\hline & $\mathrm{Chi}^{2}$ & $-0,4860$ \\
\hline & Número de observaciones & 24 \\
\hline \multirow{5}{*}{ Uruguay } & $\beta$ & $-0,6106$ \\
\hline & Prueba normal $Z$ & $-6,49$ \\
\hline & Probabilidad (valor-p) & 0,000 \\
\hline & $\mathrm{Chi}^{2}$ & $-0,7950$ \\
\hline & Número de observaciones & 24 \\
\hline
\end{tabular}

Fuente: elaboración propia con base en las estimaciones realizadas en STATA 11.

intrarregional, el crecimiento del país tiene una variación negativa en el crecimiento del $0,038 \%$.

En lo que respecta a Brasil, el modelo por país resulta ser significativo en conjunto. Si se observan los resultados por parámetros, se tiene que las variables independientes del comercio no explican los cambios en el PIB per cápita, al igual que en el caso de Argentina. La probabilidad de estas dos variables es más cercana a 1 que a cero.

Ecuación 2: Brasil $\beta$-convergencia condicional: $\operatorname{In}\left(P I B P-P I B P_{t-1}\right)=4,765-5,116 \operatorname{lnPIBP}{ }_{t-1}+$ $0,110 \operatorname{InI} A G-0,502 \ln I A I R+\varepsilon_{t}$

$$
\begin{array}{cccc}
e e(0,7652) & (0,0825) & (0,0173) & (0,0110) \\
p b(0,000) & (0,000) & (0,0000) & (0,000)
\end{array}
$$

Adicionalmente, y contrario al caso de Argentina, el parámetro $\beta_{1}(5,116)$ es negativo para Brasil, lo que significa que el país en el largo plazo estaría experimentando un proceso de convergencia en términos de PIB per cápita. Lo que no dice la ecuación es la velocidad a la que el proceso de convergencia estaría sometido. En el caso del comercio intrabloque, este resulta negativo para Brasil, tal como lo indican los coeficientes de apertura intrarregional (AIR); sin embargo, para el índice de apertura global (IAG) es positivo.

En lo atinente a la política económica de la integración, el Mercosur en general es positivo para Brasil, pues contribuye al crecimiento. No obstante, la macroeconomía de este país, en el caso específico, por ejemplo, del manejo de la tasa de interés, la inflación y el ingreso de capitales que aprecian su moneda, tiene un papel determinante sobre las tasas de crecimiento del PIB per cápita, que a veces compensan el efecto Mercosur. Otra mirada llevaría a ponderar qué ocurriría sin Mercosur, región a la que Brasil destina la mayor parte de sus exportaciones de origen industrial producidas tras la barrera del AEC. 
Por su parte, Paraguay cuenta con un modelo estadísticamente significativo a un nivel de confianza del $95 \%$. Tanto el índice de apertura global como el intrarregional explican los cambios en el PIB per cápita. Al igual que los resultados presentados para los demás países, se puede apreciar que para Paraguay también hay evidencia de $\beta$-convergencia condicional. El comercio intrarregional resulta ser inversamente proporcional ante los cambios en el PIB per cápita. En este sentido, podría decirse que es más favorable para Paraguay el comercio que sostiene con el resto del mundo que mantiene con sus socios comerciales, según los resultados obtenidos por este modelo.

Ecuación 3: Paraguay $\beta$-convergencia condicional: $\operatorname{In}\left(P I B P-P I B P_{t-1}\right)=2,001-0,2523 \operatorname{InPIBP} P_{t-1}$ $+0,1251 \operatorname{In} I A G-0,0910 \operatorname{In} I A I R+\varepsilon_{t}$

$$
\begin{array}{cccc}
\text { ee }(0,765) & (0,082) & (0,0173) & (0,011) \\
p b(0,037) & (0,034) & (0,010) & (0,069)
\end{array}
$$

Finalmente, para Uruguay el modelo en conjunto es estadísticamente significativo a un nivel de confianza del $95 \%$. El parámetro $\beta_{1}(0,6106)$ es negativo, lo que significa que el país en el largo plazo estaría experimentando un proceso de convergencia en términos de PIB per cápita.

Ecuación 4: Uruguay $\beta$-convergencia condicional: $\operatorname{In}\left(P I B P-P I B P_{t-1}\right)=5,780-0,6106 \operatorname{InPIBP}_{t-1}$ $+0,2832 \operatorname{In} I A G-0,1153 \operatorname{In} I A I R+\varepsilon_{t}$

$$
\begin{array}{llll}
\text { ee }(0,890) & (0,094) & (0,043) & (0,031) \\
p b(0,000) & (0,000) & (0,000) & (0,000)
\end{array}
$$

La conclusión de los resultados del modelo data panel, en términos de convergencia, es una para el bloque Mercosur y otra para cada país. Para el bloque no se registra "convergencia" en los PIB per cápita, y esto indicaría que no estaría obteniendo beneficios en cuanto bloque como tal. El comercio intrarregional es clave para la integración; sin embargo, no está siendo determinante el caso de cada uno de los países miembros. El intercambio comercial al interior del bloque no sería un sendero del éxito individual, pero en lo que respecta al comercio con el resto del mundo parece ser diferente.
En el caso de la relación de integración bilateral de Argentina y Brasil, los resultados del ejercicio data panel invitan a pensar en términos de búsqueda de complementación productiva y, en consecuencia, de un intercambio comercial virtuoso. Una revisión de las crisis y conflictos, planteadas a través de las "agendas" bilaterales (también, en los casos que corresponda, del bloque), muestra que la integración todavía tiene un camino largo por delante. A las "agendas" de la Profundización y la Consolidación (1996/1997) se agregó la del Relanzamiento (2000), a esta se sumó la de Reformulación (2003), y esta fue confirmada y profundizada por el Programa de Trabajo (2004/2006). A partir de este último año, todo parece estar corriendo como business as usual, o sea, sin modificaciones respecto de la historia que tiendan a aumentar los beneficios de la integración en forma neta.

\section{CONCLUSIONES}

Este trabajo mostró el impacto del Mercosur - traducido principalmente, a los efectos de la investigación empírica, en los flujos comerciales intrarregionales y globales-en el crecimiento del PIB per cápita de los países miembros, tomados como bloque e individualmente. El objetivo fue medir la convergencia del Mercosur y de cada uno de sus Estados miembro, en el entendido de que la convergencia es una de las medidas de bienestar que se utilizan para sostener si la integración arroja beneficios e incremento de bienestar para los países convocados.

El primer paso fue la fundamentación teórica de la integración, vista desde la unión aduanera y desde la revisión del criterio de convergencia. El caso es aquí particularmente interesante, dado que se trata de una integración que se encuadra en el criterio Sur-Sur. En este caso, y a diferencia de los acuerdos de tipo Norte-Norte y Norte-Sur, que cuentan con un "país locomotora", aquí no lo hay. El caso de Brasil con gran tamaño y mercado, sobre todo potencial, no lo transforma en una "locomotora", dado que necesita capital igual que el resto de los socios del Mercosur. Su PIB per cápita es mediano/bajo y sus niveles de pobreza e inequidad 
distributiva en los ingresos son elevados, además de no ofrecer un territorio cohesionado en el nivel de ingreso y riqueza. La pregunta clave aquí, entonces, es si la unión aduanera per se, tal como se ha estructurado hasta la actualidad, podría llevar a los países miembro a experimentar un mayor nivel de bienestar, tanto en lo que se respecta al bloque Mercosur como en lo que se relaciona con cada uno de los países miembros.

En cuanto a la convergencia, bajo la premisa de que los países se integran para aumentar los flujos comerciales y mejorar el bienestar, la medición de los impactos podría hacerse desde diferentes enfoques. Aquí se optó por testear el caso bajo el supuesto neoclásico, esto es, la existencia de rendimientos decrecientes en cada factor, lo cual implica que la productividad marginal del factor se reducirá con su acumulación. Este mecanismo genera una predicción de convergencia. Por su parte, la teoría del crecimiento endógeno supone que el retorno de la inversión aumenta con el stock de capital por trabajador, favoreciendo a los países más desarrollados, que en la experiencia global tienden a crecer más rápido que los pobres (De la Fuente, 2000, pp. 2-3).

El segundo paso consistió en el análisis de la evolución de la estructura productiva y patrón de comercio, con especial atención en Argentina y Brasil. Dentro de la configuración productiva y el patrón del comercio. Brasil, vía subsidios y política industrial, se posiciona como una economía en busca de ventajas comparativas en cada uno de los sectores industriales. El resto de países del bloque están direccionados hacia sectores basados en recursos naturales. Argentina, a pesar de tener una vocación hacia los productos manufacturados, ha experimentado un proceso de desindustrialización, especialmente en la década de 1990. Pese a las preferencias arancelarias otorgadas al interior del bloque, Mercosur no ha logrado estimular de forma virtuosa el comercio intrazona. Además, el comercio extrazona tiene un mayor peso en el comercio total de cada uno de los países, comparado con el intrazona.
El paso final consistió en la medición de la convergencia bajo la visión neoclásica del crecimiento. Los resultados observados tras correr el data panel ponen en evidencia la existencia de asimetrías en términos de PIB per cápita, entre los países miembros, desde la entrada en vigencia del Tratado de Asunción y hasta la actualidad.

Utilizando el modelo por efectos fijos, se observó que las regresiones no evidencian la existencia de $\beta$-convergencia en el PIB per cápita del conjunto de países, por lo cual los países tienden a la divergencia de ingresos. Adicionalmente, se estimó un modelo a partir del cual se incluye el índice de apertura global y el índice de apertura intrarregional, con el fin de determinar la existencia de $\beta$-convergencia condicional y verificar si estas variables explican los cambios en el PIB per cápita. Los resultados revelaron la no existencia de $\beta$-convergencia condicional para el conjunto de países. Si se entiende la integración como un proceso económico influenciado por las estructuras productivas de los países, cuyo patrón de comercio responde a la asignación de recursos de la estructura sectorial de la producción, se puede concluir que Mercosur no ha contribuido en el crecimiento de los países en términos de PIB per cápita.

Finalmente, los resultados obtenidos del modelo por países proveen información valiosa para el proceso del Mercosur. Mientras Argentina no obtiene beneficios del comercio intrazonal, aunque sí del comercio global, Brasil, el socio de mayor tamaño, sí logra obtener ganancias a partir del comercio intrabloque. Esto plantea posibles conflictos para esta integración, aunque sea por las manifestaciones externas vía desequilibrios comerciales, dada la situación bilateral de estructuras productivas sustitutivas y no complementarias. Este dilema debería ser tomado como el primer punto de la resolución de una integración que aparece como vacilante.

Para el caso de Brasil se puede observar que obtiene beneficios por el Mercosur, tras revisar las estadísticas de patrón y dirección del comercio. Sus socios Mercosur son destinatarios de una proporción dominante de sus exportaciones industriales. 
El país también saca beneficios concretos de la barrera proteccionista del AEC. Por su parte, los resultados conseguidos para el modelo individual de Uruguay muestran que los parámetros en conjunto no son estadísticamente significativos a un nivel de confianza del $95 \%$. Esto significa que la estructura de los datos presentados para Uruguay, en el modelo individual por país, no evidencia una relación fuerte entre el PIB per cápita y los índices de apertura global e intrarregional. En el caso de Paraguay, las variables de comercio sí explican los cambios en el PIB per cápita, pero no se encuentra evidencia de $\beta$-convergencia condicional.

Se puede concluir, además, que la voluntad política de integración entre los socios, que se mantiene como política de Estado en todos los países, se ve afectada seriamente por la distribución inequitativa del beneficio de la integración profunda, y se agudiza con las disparidades estructurales de cada país. La integración ganadora es aquella en la que no solo las economías son complementarias en producción, sino que también lo son en términos de comercio. El Mercosur debe abogar por generar corrientes de comercio acordes con las ventajas comparadas y que, por tanto, permitan la especialización de la producción. Por consiguiente, la política de complementación productiva es un eje fundamental en la consolidación del proceso de integración, pues contribuye a la especialización en determinados bienes, genera economías de escala y contribuye a aumentar la productividad, o al menos nivela en terreno para que esto suceda.

\section{REFERENCIAS}

1. Arias, C. (2008). Asimetrías en el Mercosur: un enfoque macroeconómico. En F. Masi y M. I. Terra (coords.), Asimetrías en el Mercosur: ¿impedimento para el crecimiento? (pp. 43-85). Montevideo: Red Mercosur de Investigaciones Económicas.

2. Blyde, J. (2007). Convergence dynamics in Mercosur. Journal of Economic Integration, 21(4), 784-815. https://doi.org/10.11130/jei.2006.21.4.784

3. Bouzas, R. y Pagnotta, E. (2003). Dilemas de la Política Comercial Externa Argentina. Buenos Aires: Siglo XXI, Fundación OSDE y Universidad de San Andrés.

4. Brida, J. et al. (2012). Convergencia interregional en dinámica de regímenes: el caso de Mercosur [MPRA Paper 36863]. Recuperado de https://mpra.ub.uni-muenchen.de/36863/

5. Corden, W. (1965). Recent developments in the theory of international trade (1. ${ }^{\mathrm{a}}$ ed.) Nueva Jersey: Universidad de Princeton.

6. Cresta, J. (2008). Asimetrías en el Mercosur. Un enfoque macroeconómico. En En F. Masi y M. I. Terra (coords.), Asimetrías en el Mercosur: ¿impedimento para el crecimiento? (vol. 12, pp. 41-85). Montevideo: Red MERCOSUR.

7. De La Fuente, A. (2000). Convergence across countries and regions: Theory and empirics. EIB Papers, 5(2), 25-46.

8. Díaz B., A. (2003). Apertura comercial y convergencia regional en México. Comercio Exterior, 53(11), 995-1000.

9. Karras, G. (1997). Economic integration and convergence: Lessons from Asia, Europe and Latin America. Journal of Economic Integration, 12 (4), 419-432. https://doi.org/10.11130/jei.1997.12.4.419

10. Lipsey, R. (1960). The theory of customs unions: A general survey. Economic Journal, 70. https://doi. org/10.2307/2228805

11. Mayorga , M. y Muñoz, E. (2000). La técnica de los datos de panel: una guía para su uso e interpretación. San José: Banco Central de Costa Rica. 
12. Meade, J. (1955). The theory of international economic policy. Oxford: Oxford University Press.

13. Meade, J. (1956). The theory of customs unions. Ámsterdam: North-Holland Publishing Company.

14. Montero, R. (2011). Efectos fijos o aleatorios: test de especificación. Granada: Universidad de Granada.

15. Ramón, C. (2003). Convergence or regional disparities in Argentina and Brazil: The implications for Mercosur. Londres: Royal Institute of international Affairs.

16. Sachs, J. D. y Warner, A. M. (1995). Economic convergence and economic policies. https://doi.org/10.2139/ ssrn. 1479610

17. Sala-i-Martin, X. (2000). Apuntes de crecimiento económico. Madrid: Antoni Bosch.

18. Schiff, M. y Winters, L. (2003). Regional integration and development (3. ${ }^{\mathrm{a}}$ ed.). Washington: Banco Mundial y Oxford University Press. https://doi.org/10.1596/0-8213-5078-1

19. Venables, A. (2000). Winners and losers from regional integration agreements. Londres: Londres School of Economics and CEPR.

20. Viner, J. (1950). The Custom Union Issue. Nueva York: Carnegie Endowment for International Peace.

21. Wooldridge, J. (2001). Introducción a la econometría: un enfoque moderno (vol. I). Ciudad de México: Cengage Learning Editores. 